\title{
A Reliable Path Selection and Packet Forwarding Routing Protocol for Vehicular Ad hoc Networks
}

\author{
Irshad Ahmed Abbasi ${ }^{1,2^{*}}$, Adnan Shahid Khan ${ }^{1}$ and Shahzad Ali $^{3}$
}

\begin{abstract}
Vehicular ad hoc networks (VANETs) have earned a gigantic consideration in the recent era. Wide deployment of VANETs for enhancing traffic safety, traffic management, and assisting drivers through elegant transportation system is facing several research challenges that need to be addressed. One of the crucial issues consists of the design of scalable routing algorithms that are robust to rapid topology changes and frequent link disconnections caused by the high mobility of vehicles. In this article, first of all, we give a detailed technical analysis, comparison, and drawbacks of the existing state-of-the-art routing protocols. Then, we propose a novel routing scheme called a Reliable Path Selection and Packet Forwarding Routing Protocol (RPSPF). The novelty of our protocol comes from the fact that firstly it establishes an optimal route for vehicles to send packets towards their respective destinations by considering connectivity and the shortest optimal distance based on multiple intersections. Secondly, it uses a novel reliable packet forwarding technique in-between intersections that avoids packet loss while forwarding packet due to the occurrence of sudden link ruptures. The performance of the protocol is assessed through computer simulations. Simulation outcomes specify the gains of the proposed routing scheme as compared to the earlier significant protocols like GSR (Geographic Source Routing), GPSR (Greedy Perimeter Stateless Routing), E-GyTAR (Enhanced Greedy Traffic Aware Routing), and TFOR (Traffic Flow-Oriented Routing) in terms of routing metrics such as delivery ratio, end-to-end delay, and routing overhead.
\end{abstract}

Keywords: Multiple intersections, Position-based routing, Optimal route, Forwarding

\section{Introduction}

The immense growth of automobiles and irregular behavior of drivers on the road cause traffic congestion, accidents, wastage of fuel, and loss of precious lives, which makes the existing transportation system inefficient. To direct these challenges, a new research field called as Intelligent Transportation System (ITS) has been proposed. It applies a combination of multiple promising technologies of automobiles and transportation system in order to enhance security, safety, effectiveness of transportation systems, vehicle control, and provision of latest mobile services and applications to the on-road public by advancing traffic management system. In ITS, developing vehicle to vehicle and vehicle to infrastructure communication is an

\footnotetext{
* Correspondence: irshad_upesh@yahoo.com

1 Department of Computer Science and Information Technology, Universiti Malaysia Sarawak (UNIMAS), 9300 Kota Samarahan, Malaysia

${ }^{2}$ Department of Computer Science, Faculty of Science and Arts at Balgarn, University of Bisha, P.O. Box 60, Sabt Al-Alaya 61985, Kingdom of Saudi Arabia Full list of author information is available at the end of the article
}

outstanding challenge to ITS industry. Thus, the US Federal Communication Commission has approved $75-\mathrm{MHz}$ spectrum at $5.9 \mathrm{GHz}$ for dedicated short-range communications (DSRC) [1-3] for the successful deployments of WLAN technologies for making vehicular ad hoc networks (VANETs) a reality.

From the last few years, inter-networking over VANETs has been achieving a massive momentum. Realizing its intensifying significance, the academic research society, major car manufacturers, and governmental institutes are making efforts to develop VANETs. Various significant projects are initiated by different countries and famous industrial firms such as Daimler-Chrysler, Toyota, and BMW for inter-vehicular communications. Some of these prominent projects include CarTALK2000 [4], Car-to-Car Communication ConsortiumC2CCC [5], Advanced Driver Assistance Systems (ADASE2), California Partners for Advanced Transit and Highways (California PATH) [6], FleetNet [7], DEMO 2000 by Japan Automobile Research 
Institute (JSK) [8], Chauffeur in EU [9], and Crash Avoidance Metrics Partnership (CAMP) [10]. These developments are the key steps towards the recognition of intelligent transportation services.

VANETs are a particular offshoot of MANETs. Due to the fast motion of the vehicles, they have more rapidly and dynamically changing topology as compared to MANETs. But the mobility of the vehicular nodes is restricted by predefined roads layout. Speed limits, congestion level, and traffic control systems like traffic lights and stop symbols also restrict the vehicular node velocities. Additionally, future vehicular nodes can be provided with larger transmission ranges, broad onboard storage and sensing capabilities, and rechargeable energy sources. Unlike MANETs, VANETs are rich in storage and processing power capabilities which make them flexible and make them more compatible of doing computationally intensive tasks [11-15].

There are various technical challenges for the design of efficient vehicular communications. One of the most critical challenges of the vehicular ad hoc network is to develop a scalable and reliable multi-hop routing protocol that is capable of providing an optimal route for forwarding packets towards the destination. Guaranteeing a stable, robust, optimal, and reliable multi-hop routing mechanism over VANETs is a fundamental move towards the realization of efficient vehicular ad hoc communications. Many emerging applications in vehicular communication require the assistance of multi-hop communications. One of them is onboard active safety mechanisms which help drivers to avoid collisions and provide coordination at crucial positions like highway entries and city intersection/ junctions [16]. With the help of safety system information about roads like real-time traffic congestion, traffic accidents, road surface conditions, or high-speed levy can be smartly disseminated. This helps in avoiding the road congestion. It also largely reduces vehicle accidents and helps in saving many precious lives. In addition, inter-vehicular communication can provide comfort and infotainment applications. These consists of information about weather conditions, locations of gas stations and restaurants, e-commerce, and infotainment applications like accessing the internet, downloading music, and content delivery $[14,17-20]$.

The characteristics like non-uniform distribution of vehicles on the road, large size network, high mobility due to the high speed of the vehicles, frequently changing topology, and disruption of communication due to obstacle hindrance make routing of data in the vehicular ad hoc network more challenging. Majority of existing routing techniques are incapable of deciding optimal routes because of inefficiently incorporating aforementioned characteristics of VANETs. One of the problems with these routing protocols is that they often relay the packet towards destination using those streets that do not contain enough vehicular density. As a result, the packet meets a local optimum situation. Local optimum is a situation when a forwarding node is unable to locate the next neighbor because of lack of traffic density on the street. The node keeps the packet in its buffer for a longer time. If packet stays a longer time in the buffer, its time to live field expires and is eventually discarded. This degrades the network performance in terms of end-to-end delay and packet delivery ratio. Designing a routing protocol capable of solving such issues is critical and our proposed protocol intends to overcome this issue by selecting multiple connected streets that contain high vehicular density based on multiple intersections. Our findings focus on those multiple intersections or streets that provide optimal routing path based on closest distance to destination and contain high traffic through which packet can be easily relayed towards the destination. Secondly, besides optimal path based on multiple streets, we also propose a reliable packet forwarding strategy based on link stability and predicted packet propagation time between packet carrier node and next candidate forwarding neighbor to overcome packet loss due to unstable links.

The major contributions of this research are as follows:

1. Presented technical analysis, comparison, and drawbacks of earlier significant position-based routing schemes in VANETs.

2. Provided the significance of dynamic multiple intersection selection mechanism and limitations of earlier dynamic intersection selection mechanism pertaining to the city environment. Presented a novel routing scheme for city environment that is based on multiple intersections selection mechanism, which decides the best multiple streets to forward the packet towards the destination based on optimal distance and traffic density. To the best of our knowledge, this is the first time multiple intersection selection-based routing issue being thoroughly studied.

3. Along with multiple intersections selection mechanism, we introduced a novel reliable forwarding technique that considers link duration time and expected packet delivery time to overcome the packet loss issue due to high mobility and intermittent connectivity of vehicular nodes.

4. We provided an analysis and compared the performance of our routing scheme with existing approaches (GSR, GPSR, E-GyTAR, and TFOR) using ONE simulator. Simulation results indicate the benefits of the proposed routing strategy as compared to the existing protocols like GSR, GPSR, E-GyTAR, and TFOR in terms of packet delivery ratio, end-to-end delay, and routing overhead. 
The remaining paper is structured as follows. In Section 2, we briefly present methods used in our study. In Section 3, we summarize the earlier routing protocols by highlighting their drawbacks which act as a source of persuasion for our research. Proposed routing strategy is presented in Section 4. Then, we present the performance evaluation based on extensive simulations in Section 5. In Section 5, we study and recapitulate the evaluation results. Finally, Section 6 concludes the paper.

\section{Methods}

For the performance evaluation of the proposed protocol, extensive simulations were used to compare the performance of our protocol with other state-of-the-art routing protocols. For the performance evaluation, it is vital to use a state of the art simulation environment capable of performing the simulations reliably and efficiently. In the research community for VANETs, the most versatile and well-trusted simulation environment is provided by the ONE simulator [21]. In this study, we used ONE simulator for all the simulations. Another important aspect regarding the performance evaluation of VANETs is the mobility model to be used. We utilized SUMO (simulation of urban mobility) [22] for generating the realistic vehicular mobility patterns to be used for the performance evaluations. SUMO is a microscopic road traffic simulation package, and the mobility traces generated by SUMO incorporates all essential characteristics of the city environment. More detail about the simulation environment and vehicular mobility patterns is provided in the simulation setup section. The proposed protocol was implemented in SUMO, and similarly, all the other considered routing protocols were also implemented. The performance metrics used for the comparison of the proposed protocol with the existing protocol were packet delivery ratio, end-to-end delay, and routing overhead. The performance was evaluated based on the considered performance metrics, and a variety of results were obtained and presented in this study.

\section{Related work}

The existing routing protocols (like Dynamic Source Routing (DSR) [23], Ad hoc On-Demand Distance Vector (AODV) [24], and Optimized Link State Routing (OLSR) [25]) that were originally proposed for mobile ad hoc networks are ineffective for VANETs $[1,11,12,21,26-$ 28 . These routing techniques consider the address of mobile nodes while discovering and maintaining end-to-end routing path in between the source node and destination. In vehicular ad hoc communication, the irregular distribution and high mobility of vehicular nodes frequently break the routing paths which make it hard to maintain and find routes. Consequently, these routing techniques generate high control overhead which degrades network performance
$[1,4,6,11,12,14,18,29-34]$. The other class of routing which considers a geographical position of mobile nodes instead of address is suitable for vehicular ad hoc communication [11, 26, 27, 31, 32, 34, 35]. This class is more enviable for VANETs because of the following facts. Firstly, in the near future, vehicles will be implanted with navigation systems and Global Positioning Systems (GPS); thus, position-based routing class accomplishes colossal achievement in vehicular communication. Secondly, this class of routing is stateless; there is no need to maintain accomplished routing paths in between the source and target nodes; hence, this class is exceedingly scalable and very robust against high mobility which frequently changes network topology [11, 21, 27, 31, 34].

VANETs can be deployed into two different environments: (1) city/urban and (2) highway. City environment consists of intersections. Intersections are the points where two or more roads meet each other. On the other hand, a highway environment contains no intersection $[1,21,33,36]$. In a city environment, the different sequence of intersections can play an important role in providing the shortest distance from source to destination. In the existing literature, some protocols are dynamic intersection selection based, some are static intersection selection based, and some having no intersection selection mechanism at all. Figure 1 provides the classification of the protocols. Below, we provide a brief technical description of existing significant position-based routing protocols that are designed for the city environment.

Greedy Perimeter Stateless Routing (GPSR) [28] is designed for handling routing issues in the highway environment. In a highly dense scenario, it performs well. It has two modules that are greedy module and perimeter module. In the greedy module, a vehicular node forwards the packet to one of its one-hop neighbors that is the closest among its one-hop neighbors and itself to the destination. The greedy module meets the local maximum if the packet carrier node has no one-hop neighbor that is close to the destination than itself. Perimeter module is used to handle the local maximum situation. The perimeter module includes two mechanisms, the graph planarization, and the right hand rule. The perimeter module induces long delays in dispatching packets from the source to the target. It also creates routing loops in the network, and unaware of obstacles [37], which makes it difficult to work in city scenarios. Furthermore, the graph planarization partitions the network in the city scenarios due to obstacles and degrades its performance further $[11,12]$.

Geographic source routing (GSR) [37] is a positionbased routing protocol designed for urban scenarios. It uses position awareness with network topological awareness. It accomplishes the shortest route between the 


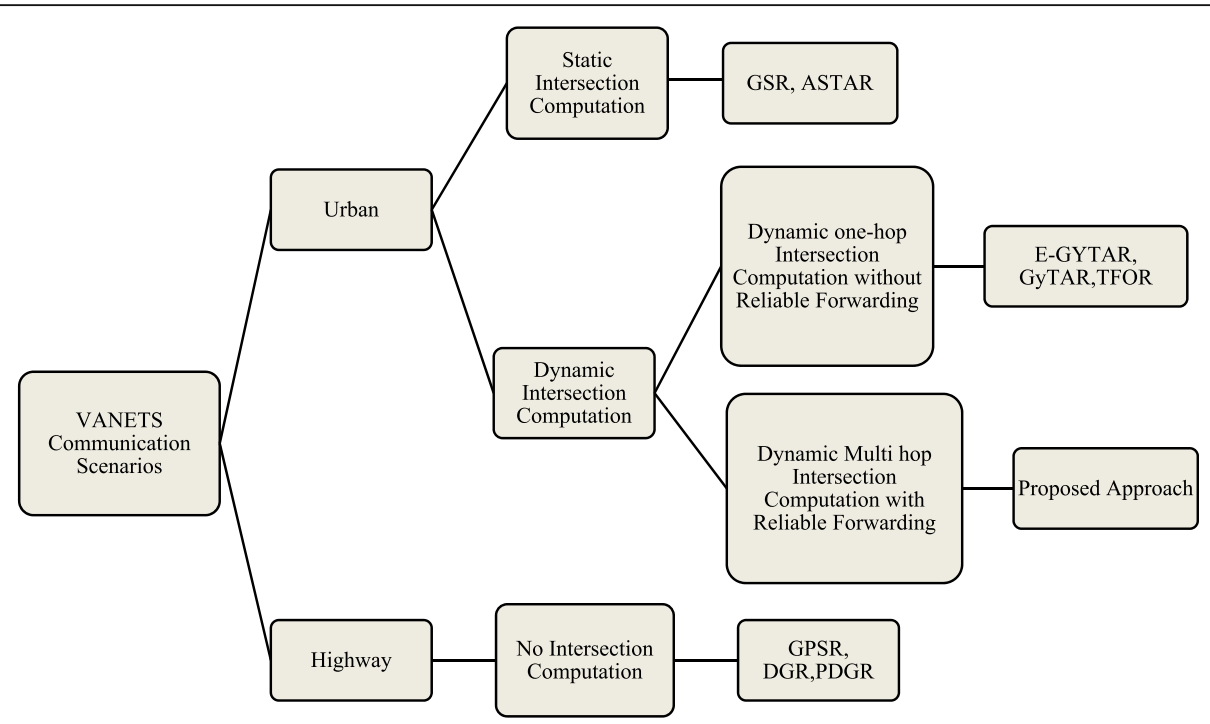

Fig. 1 Classification of position-based routing protocols

source and target node using Dijkstra's algorithm. The shortest route accomplished by GSR with the help of digital map consists of a sequence of intersections through which packet moves towards the destination. The packets are sent to the destination based on greedy forwarding. Greedy forwarding causes local optimum. GSR recovers from local optimum using carry and forward approach. While accomplishing the shortest path, GSR does not consider traffic density between intersections. In low-traffic density scenarios along a preselected route, establishing an end-to-end connection is very hard which degrade the performance of network [14]. Also, in GSR, greedy forwarding suffers from sudden link ruptures.

The Greedy Perimeter Coordinator Routing (GPCR) [38] accomplishes routing path without considering a map. It is composed of restricted greedy forwarding and a repair strategy. In restricted greedy forwarding, a packet carrier node prefers to forward the packet to a node that is located nearest to the intersection or at the intersection. The coordinator node is responsible to choose next-street for relaying packet towards the destination. GPCR uses repair strategy to overcome the local optimum problem. The repair strategy includes perimeter mode without considering graph planarization. It assumes that topological graphs are naturally planner in city environment. Computing graph planarization induces network disconnections which degrade network performance. GPSR does not consider low vehicular density cases while routing [1]. It is not a traffic aware routing protocol $[11,14]$, and it does not consider the status of link while forwarding the packets that may result in excessive packet loss.

The Anchor-Based Street and Traffic Aware Routing (A-STAR) [30] is a traffic aware routing protocol as compared to GPSR and GPCR. It has two main distinctive characteristics. Firstly, for traffic awareness, it uses statically or dynamically rated maps which assist it to discover routes that have a higher number of vehicles. Secondly, to overcome local optimum problem, it has a novel local recovery strategy which is better than those of GSR and GPSR [14]. The routing path accomplished by A-STAR on the basis of anchors may not be the optimal path, which may induce higher end-to-end delay $[1,11,14]$. It has no mechanism to overcome link ruptures as well that may result in packet loss.

Greedy Traffic Aware Routing Protocol (GyTAR) [14] was designed specifically for city environments. GSR and A-STAR select intersection statically while GyTAR selects intersection dynamically. It has three mechanisms: (a) intersection selection mechanism, (b) Infrastructure Free Traffic Information System (IFTIS), and (c) an improved greedy forwarding in-between intersection. It assigns weights to each neighboring intersection on the basis of the distance to destination and traffic density. The intersection that has highest traffic density and shortest distance to the destination is decided as next intersection, and thereby, packets are routed towards the destination. At low traffic density, its intersection selection mechanism sometime meets local optimum in city environment which degrades its performance [1]. Moreover, GyTAR has no mechanism to minimize packet loss due to link ruptures that may result in packet loss.

Enhanced Greedy Traffic Aware Routing (E-GyTAR) [1] is an enhanced version of GyTAR. It chooses junction on the basis of directional density on multilane streets. Non-directional density is also helpful in relaying packet from source to destination. Hence, it misses some shortest path while routing data [11]. Its forwarding 
strategy also suffers from sudden links rupture problem due to high mobility of the vehicles.

Directional geographic source routing (DGSR) [36] is an enhanced version of geographic source routing (GSR) with directional forwarding strategy. In this routing scheme, the source vehicular node uses location services $[39,40]$ to acquire the position of destination vehicle. It establishes the shortest path from source to destination using Dijkstra Algorithm. The shortest path consists of intersections which are ordered sequentially. The packets from source vehicular node follow the sequence of intersections to reach destination. If packet meets local optimum, DGSR uses carry and forward approach to overcome local optimum problem. However, this protocol does not consider the status of link while forwarding. Therefore, in case of high mobility of vehicular nodes, it suffers from packet loss due to link raptures.

Enhanced Greedy Traffic Aware Routing ProtocolDirectional (E-GyTAR-D) [36] is an enhanced version of E-GyTAR [1] with directional forwarding. It consists of two mechanisms: (i) intersection selection and (ii) directional greedy forwarding strategy. It uses location services to get the position of destination node. It selects intersections on the basis of directional traffic density and shortest distance to the destination. It forwards packets in between intersection using directional greedy forwarding. Simulation outcomes in realistic urban scenarios show that E-GyTAR-D outperformed GSR and DGSR in terms of packet delivery ratio and end-to-end delay.

Traffic flow-oriented routing protocol (TFOR) [11] is a recently proposed technique which consists of two modules: (a) an intersection selection mechanism based on traffic flows and the shortest routing path and (b) a forwarding strategy based on two-hop neighbor information. It accomplishes shortest optimal path based on shortest distance to the recipient node and vehicular traffic density. Simulation results show that TFOR outperforms E-GyTAR and GyTAR in terms of packet delivery ratio and end-to-end delay. Table 1 shows the comparative characteristic of all the aforementioned routing protocols.

A majority of the aforementioned protocols (like GSR, DGSR, GPSR, DGR, PDGR, PDVR, and GPCR) do not consider traffic density while accomplishing routing path. Traffic density is a major source of providing connectivity. Consequently, these routing protocols relay the packets towards destination through those city streets which have low traffic density or connectivity. As a result, packets meet frequently local optimum and this leads to a decrease in packet delivery ratio. This drawback can be overcome by having a mechanism that is capable of giving timely information about city street traffic density. Although, few of the aforementioned routing schemes such as E-GyTAR, GyTAR, E-GYTAR-PD, and TFOR are traffic-aware, all of these approaches prove to be incompetent in making full use of the real-time traffic density. The intersection selection mechanism of these routing protocols decides sometime those streets that lead to a local optimum problem regardless of the availability of effective streets which may overcome such problem. In such a situation, the probability of packet loss increases. It also increases end-to-end delay because the packets are carried in the buffer for a long time. Consequently, they prove to be ineffective in relaying packets from source to destination. Also, in between successive intersections, the aforementioned routing protocols use different types of forwarding strategies like simple greedy forwarding, restricted greedy forwarding, directional greedy forwarding, and improved greedy forwarding. However, their forwarding approaches do not consider link status and link lifetime between packet carrier node and next candidate neighbor forwarding node. Therefore, these strategies miss some appropriate candidate neighbor nodes which are stable and reliable for packet forwarding. Hence, all aforementioned routing suffer from sudden link rupture problem due to the high mobility of the vehicular nodes.

In this research work, we devise a routing scheme that addresses the aforementioned drawbacks of existing protocols. The proposed routing scheme is capable of accomplishing optimal routing paths in city scenarios. The scheme is envisaged to function well for various kinds of vehicular communication applications by assuring user connectivity. These appliances consist of road safety services such as coordinated communication of two vehicles, managing flows of traffic, triggering driving-related alerts such as traffic congestion alerts, road situation alerts, and accident warnings. The other appliances include finding locations of petrol stations and restaurants, accessing internet, downloading music, and playing games.

\section{Reliable Path Selection Packet Forwarding (RPSPF) Routing Protocol}

\subsection{Problem formulation}

In this work, we considered the protocols that are based on one-hop dynamic intersection selection mechanism and forwarding strategy in between intersections for forwarding packets. These protocols include GyTAR, E-GyTAR, and TFOR. All these routing protocols choose dynamically one intersection at a time on the basis of vehicular traffic density and shortest distance to the destination while relaying a packet towards the destination. However, their one-hop dynamic intersection selection mechanisms have some limitations. As an example, we imagine one of the possible situations revealed in Fig. 2.

Assume $\mathrm{S}$ is the source vehicular node which is at intersection $I_{1}$. It intends to forward the packet to 


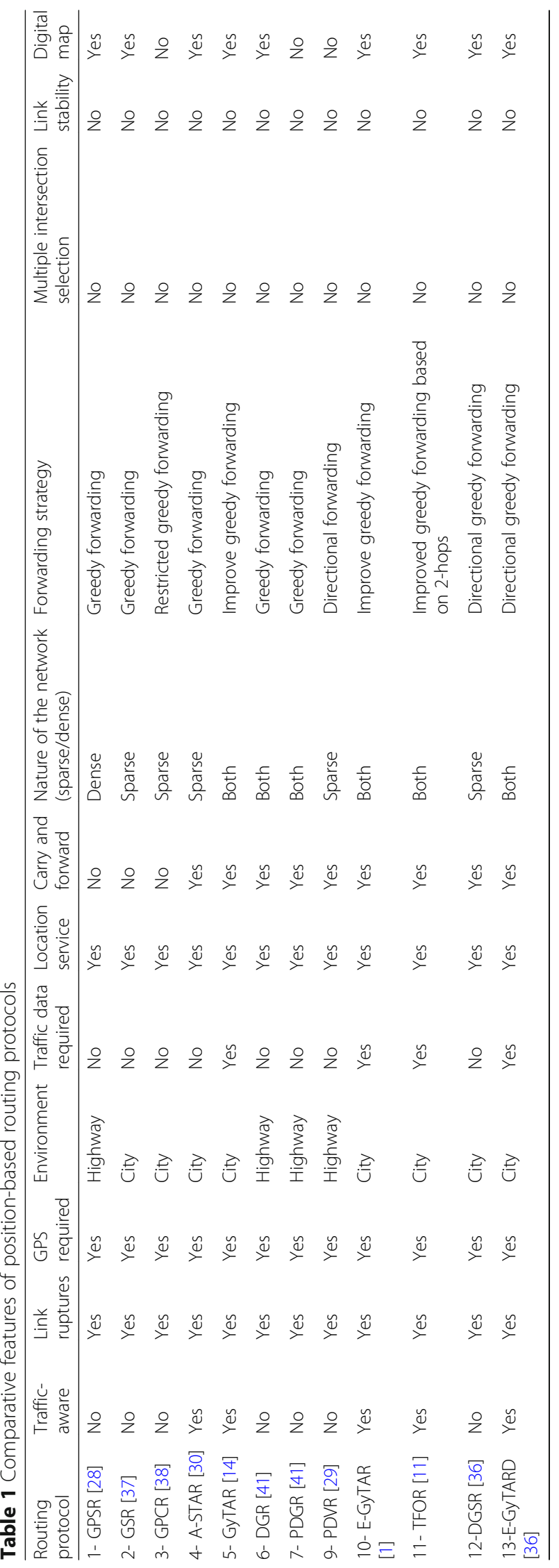




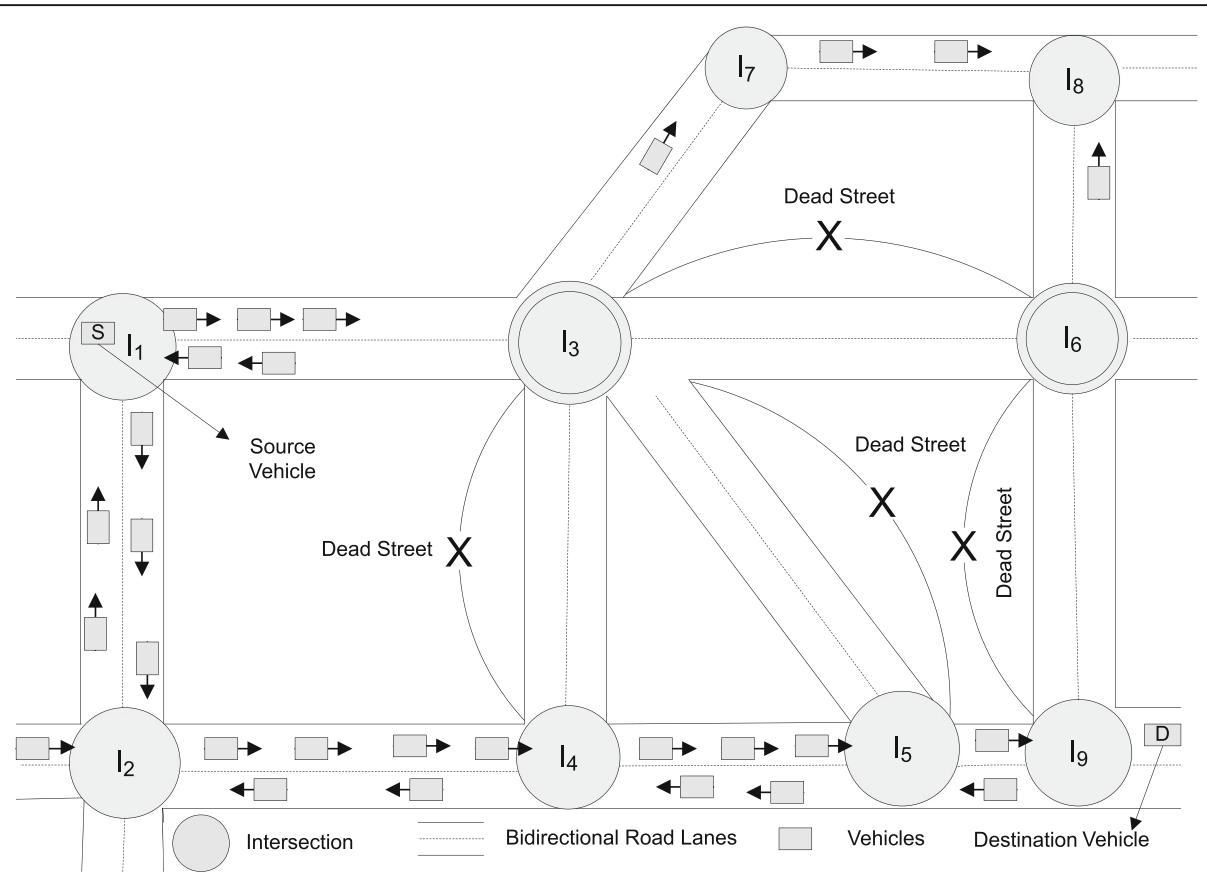

Fig. 2 The problem scenario. Each circle represents a junction. Double lines represent bidirectional two-lane roads, and the small rectangular box with arrow represents vehicular node moving in the direction of the arrow, $\mathrm{S}$ represents source vehicle, and D indicates the destination

destination vehicular node D. Current dynamic intersection selection-based routing protocols like E-GyTAR, GyTAR, and TFOR select next intersection based on vehicular traffic density and shortest distance to the destination; therefore, their intersection selection mechanism will bound them to choose $I_{3}$ as the next intersection. At $I_{3}$, TFOR, GyTAR, and E-GyTAR will be inept to choose next appropriate intersection through which packet can be relayed towards the destination. This is because the next shortest path offering roads/streets that direct towards destination have no traffic density for dispatching packet towards destination. When the packet reaches $I_{3}$, all the aforementioned protocols are unable to choose next streets because from $I_{3}$ to $I_{6}$, there is no vehicular density. Likewise, $I_{3}$ to $I_{5}$ and $I_{3}$ to $I_{4}$, there is no vehicular traffic density. Each of these streets is out of traffic, and there is no vehicular traffic density for routing packet further. This improper selection of intersections degrades the network performance as it decreases the packet delivery ratio and increases end-to-end delay regardless of the availability of the optimal path $\left(I_{1}-I_{2}-I_{4}-I_{5}-I_{9}\right)$ with rich vehicular traffic density. Consequently, all these protocols can prove to be inefficient in intersection selection mechanism because of consideration of just immediate intersection while selection of next intersection. Furthermore, their current intersection selection mechanism is very limited to move the packet progressively closer to the destination due to the consideration of just the immediate intersection from the current intersection. This increases the probability of incurring a dead street (street without packet carriers/vehicles) along a selected path to the destination due to consideration of just the immediate intersection.

The second problem with these routing protocols is that their forwarding strategies suffer from sudden link rupture problem due the high mobility of the vehicular nodes which cause packet loss. Existing forwarding strategies include greedy forwarding [28], restricted greedy forwarding [38], improved greedy forwarding [14], directional greedy forwarding [41], and predictive directional greedy forwarding [41]. None of these forwarding strategies have mechanism which is capable of handling sudden link rupture. Figure 4 in Section 4.2.3 describes the limitation of existing forwarding strategies without considering link stability mechanism.

We need a routing protocol that provide an efficient intersection selection mechanism which selects intersections by considering connectivity and a reliable forwarding mechanism which overcomes packet loss due to sudden link ruptures so that packet delivery ratio is maximized and routing overhead and end-to-end delay are minimized. We propose a novel routing strategy called Reliable Path Selection and Packet Forwarding Routing Protocol (RPSPF), which selects the next junction by considering multiple intersections based on the shortest 
path and traffic density and forwards packet using reliable forwarding strategy for tackling aforementioned circumstances.

\subsection{RPSPF protocol}

In this section, we explain the basic work of the proposed protocol.

\subsubsection{Protocol assumptions}

RPSPF is an intersection-based geographic routing protocol. It has certain assumptions similar to the assumptions made in $[1,11,14,36]$. A vehicle locates its position using GPS. Location service like GLS (grid location service) [42] can be used to locate the location of the destination vehicle. Each vehicle is equipped with an onboard navigation system that gives the position of neighboring intersections and valuable city street level awareness with the assistance of preloaded digital maps. Furthermore, it is also assumed that each vehicle is aware of its speed and direction. We also assume that each vehicle is aware of vehicular traffic density in between intersections which can be accomplished by deploying traffic sensors besides intersections or a distributed mechanism for road traffic density estimation apprehended by all the vehicles [43].

RPSPF comprises of two phases: (i) dynamic multiple intersection selection mechanism and (ii) reliable greedy forwarding mechanism between the intersections. The detailed description of both of these phases is given below.

\subsubsection{The dynamic multiple intersections selection mechanism}

RPSPF routing scheme employs anchor-based routing approach with city streets awareness like other routing routing schemes such as E-GyTAR [1], GyTAR [4], and TFOR [11]. It applies street map topology to route data packets between vehicular nodes. The foremost difference between our routing scheme and the existing routing schemes is its intersection selection mechanism and reliable forwarding mechanism in between intersections. RPSPF choose the next suitable intersection by taking into consideration the next two of the immediate intersections dynamically from the current intersection on the basis of vehicular density and shortest curve metric distance to the destination. Now, this raises a very important question about our proposed routing scheme that is, why two intersections are significant to consider instead of three, four, and so on? This query is addressed in detail in Section 5.2.4. Consideration of two intersections reduces the likelihood of incurring connectivity problem that prevailed in earlier intersection selection techniques. It also minimizes the possibility of facing those streets that contain no packet carriers or vehicular nodes along a chosen route to destination. Such streets are called dead streets. Hence, RPSPF uses those streets that are rich in connectivity and moves packets progressively closer to the destination in comparison to earlier routing schemes. While selecting the next two-hop neighbor intersection, the sender or intermediate vehicle uses digital city street map and finds the locations of two-hop neighboring intersections. It assigns weight to each of candidate two-hop neighbor intersections based on traffic density and curve metric distance of candidate intersections to the destination using Eq. 1 . The two-hop neighbor intersection with the highest weight is chosen as the next destination intersection. Algorithm 1 is used to allocate weight to each of candidate intersection.

$$
\text { Weight }=H_{1} \cdot\left(W_{j}\right)+H_{2} \cdot\left(W_{k}\right), \ldots
$$

where

$$
W_{j}=\alpha \times\left(1-\mathrm{Dp}_{1}\right)+\beta \times \mathrm{TD}_{1}
$$

and

$$
W_{k}=\alpha \times\left(1-\mathrm{Dp}_{2}\right)+\beta \times \mathrm{TD}_{2}
$$

$W_{j}$ is the weight for candidate one-hop neighbor intersection of the current intersection; $\mathrm{Dp}_{1}$ is closeness of candidate neighbor intersection with respect to destination. $\mathrm{TD}_{1}$ is the traffic density between current intersection and next neighbor intersection. $W_{K}$ is the weight for candidate neighbor intersection of the one-hop neighbor intersection; $\mathrm{Dp}_{2}$ is closeness of candidate two-hop neighbor intersection w.r.t destination. $\mathrm{TD}_{2}$ is the traffic density between the one-hop neighbor intersection and its next neighbor intersection. Alpha $(\alpha)$ and beta $(\beta)$ are the weighting factors for distance and traffic density respectively between the intersections. By adjusting the value of $\alpha$ and $\beta$, we can make tradeoff between distance and traffic density when selecting next intersection. Traffic density is an important source of giving connectivity for dispatching packet towards the destination. $H_{1}$ and $H_{2}$ are the weighting factors for candidate one-hop neighbor intersection and two-hop neighbor intersection, respectively. An adjustment in the value of $H_{1}$ and $H_{2}$ can make tradeoff between the importance of candidate one-hop neighbor intersection and two-hop neighbor intersection.

In the aforementioned algorithm 1, lines 1 to 10 state the parameters that are utilized in our algorithm to allocate weights to neighbor intersections of the candidate intersection. Line 12 checks if candidate one-hop neighbor has next neighbor intersection that leads to the 


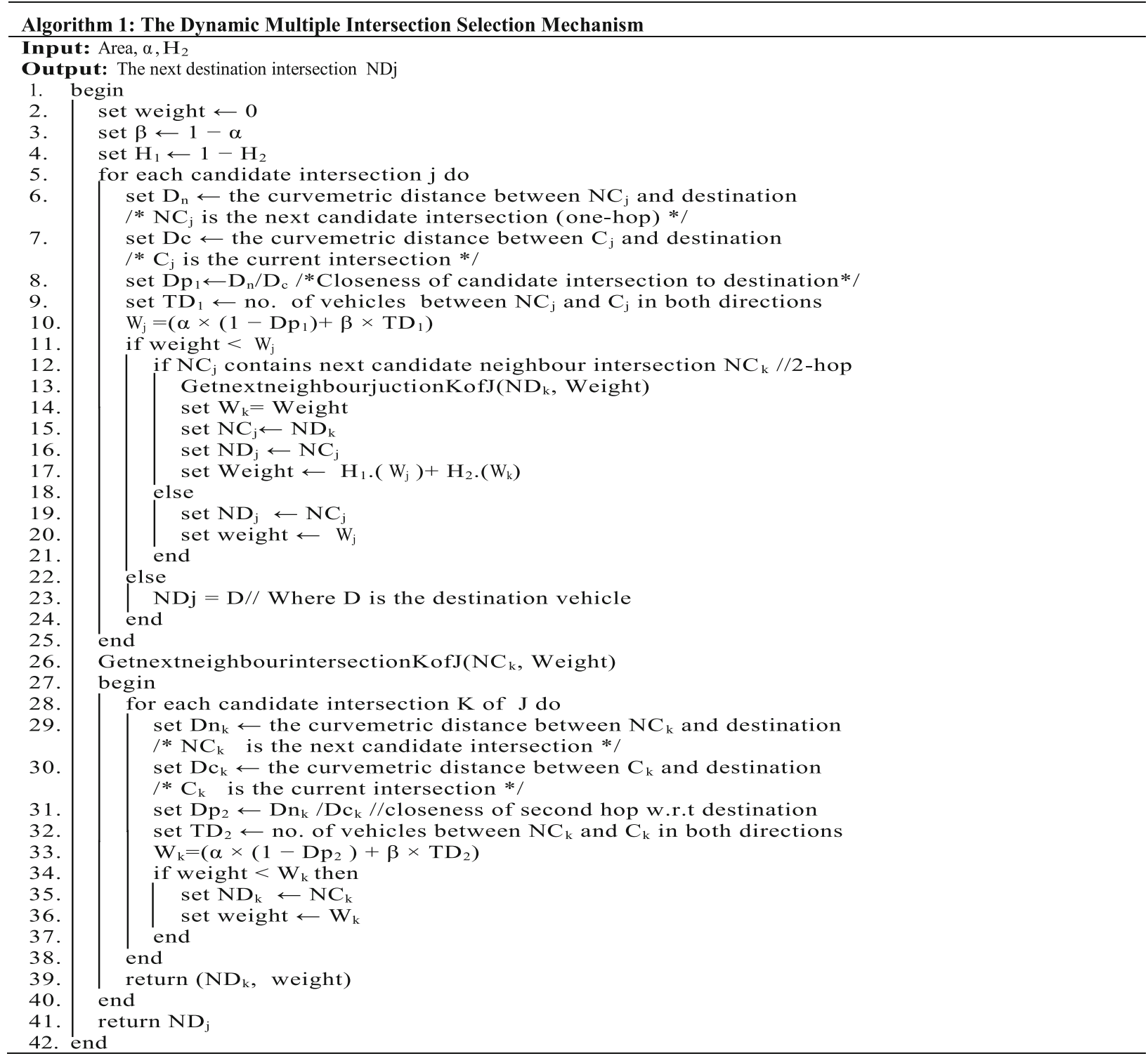

destination then algorithm call procedure mentioned in line 13 which uses line 26 to 40 to allocate weight to each of two-hop neighbor intersections. Line number 17 calculates the weight of the two-hop intersection, and the intersection having the maximum weight will be chosen as the next two-hop candidate neighbor intersection through which packet is relayed towards the destination. Any candidate two-hop neighbor intersection that is the closest to the destination and provides higher traffic density will be selected as next destination intersection through which packet moves towards the destination.

In order to understand the working of RPSPF, let us consider the scenario presented in Fig. 3. $S$ is the source vehicle which is present at intersection $I_{1}$. It uses RPSPF for dispatching packet towards the destination D. In this scenario, current intersection $I_{1}$ contains four candidate two-hop neighbor intersections through which packets can be relayed towards the destination. These intersections are $I_{4}, I_{5}, I_{6}$, and $I_{8}$. The intersection selection mechanism in RPSPF will allocate weights to each of two-hop neighbor intersections of $I_{1}$ on the basis of vehicular traffic density and the shortest curve metric distance to the destination. There is a higher concentration of vehicular traffic along the streets that are connecting $I_{1}$ to $I_{4}$ as compared to $I_{1}-I_{5}, I_{1}-I_{8}$, and $I_{1}-I_{6}$. Accordingly, RPSPF will allocate more weight to $I_{4}$ as compared to $I_{5}, I_{6}$, and $I_{8}$ and it will be selected as the 


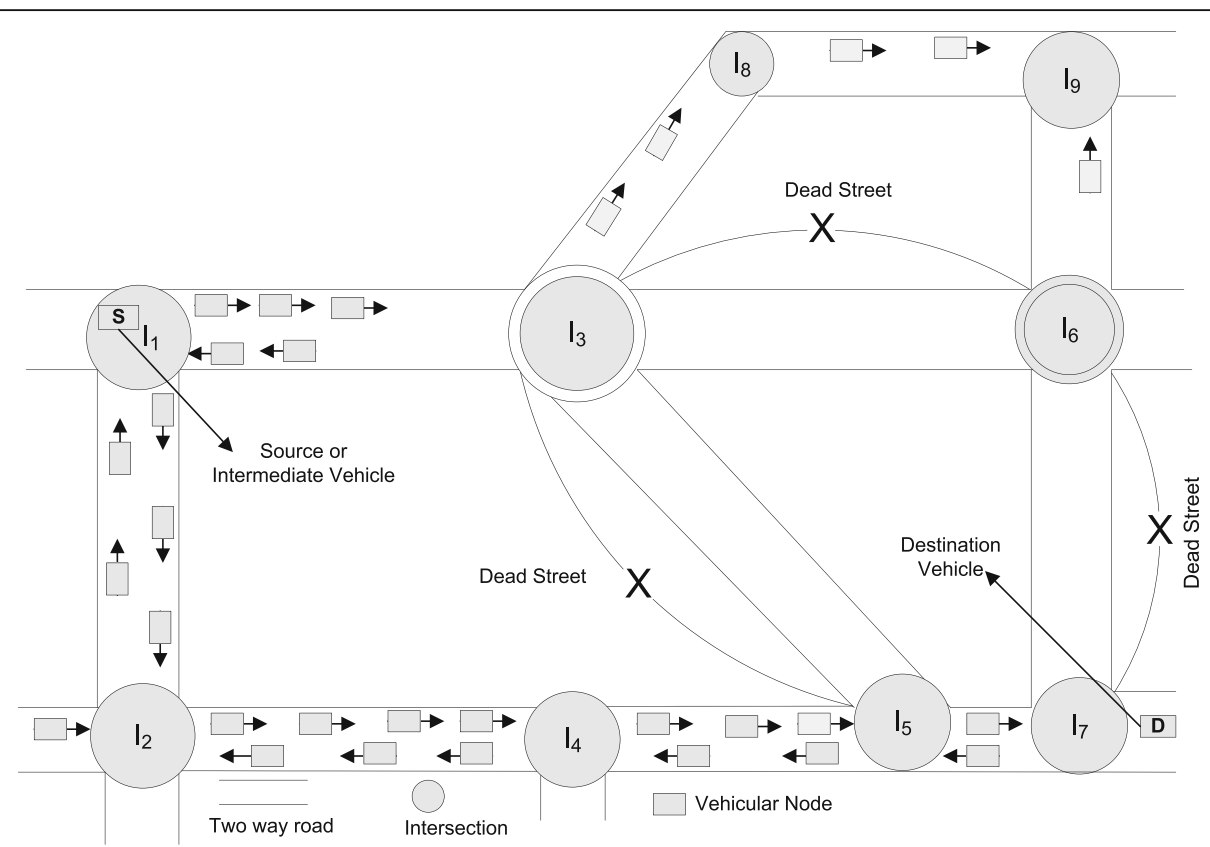

Fig. 3 Working of RPSPF protocol. Each circle represents a junction. Double lines represent bidirectional two-lane roads, and the small rectangular box with arrow represents vehicular node moving in the direction of the arrow, $\mathrm{S}$ represents source vehicle, and $\mathrm{D}$ indicates the destination

next destination intersection. In this manner, it will overcome the precincts of earlier routing approaches like TFOR, GyTAR, and E-GyTAR. Since in earlier routing schemes, $I_{3}$ would have been chosen. This is because all these routing schemes are based on one-hop intersection selection mechanism, which is the sub-optimal option in this scenario. The packet would have been trapped in local optimum because after choosing $I_{3}$, intersections $I_{5}$ and $I_{6}$ have lack of traffic density as these are along dead streets which contain no vehicular node for carrying packet towards the destination. Our routing scheme route the packet from current intersection $I_{1}$ to $I_{4}$ through $I_{2}$, and in this manner, it will dispatch the packet towards the destination. Consequently, RPSPF will move packet successively closer towards the destination beside the city streets where there are plenty of vehicles to give connectivity.

\subsubsection{Reliable forwarding mechanism between intersections}

The forwarding strategies that are used by TFOR, GyTAR, E-GyTAR, GPSR, and GSR suffer from a sudden link rupture problem due to high mobility in VANETs. Figure 4 shows a scenario where GyTAR and E-GyTAR prove to be inefficient. If the forwarding vehicle $F$ is using GyTAR, E-GyTAR, GPSR, or GSR, the forwarding strategies used in these routing protocols will compel $F$ to select $B$ as the next hop for forwarding packet as B is the closest to the destination and moving in the direction of the destination. But if $\mathrm{F}$ forwards packet at time $t_{1}$ to $\mathrm{B}$ and at the same time $B$ leaves the range of $F$ due to high speed, then the packet will not be delivered to $B$ and it will be lost. In general, we can say that if packet delivery time is greater than the link duration time between forwarding node and the next neighbor node, then packet cannot be delivered to the next neighbor node. This will decrease the packet delivery ratio and thus reduces the throughput of the network. We try to overcome this problem by taking into account link duration time between two mobiles nodes and the expected packet delivery time.

\subsubsection{Link duration time}

If the parameters like speed, position, radio propagation range, and direction that are related to the motion of two neighboring vehicular nodes are known, then the time duration for which these two nodes will remain in contact can be determined [44]. Consider two vehicular nodes $j$ (packet carrier or forwarding node) and $k$ (next candidate packet receiving neighbor node) inside the transmission range of each other. Let us assume the $\left(X_{j}\right.$, $Y_{j}$ ) be the coordinates of the location for vehicular node $j$ and $\left(X_{k}, Y_{k}\right)$ be the coordinates of vehicular node k. Let $V_{j}$ and $V_{k}$ be the velocities, $\left(\theta_{j}, \theta_{k}\right)$ be the directions of movement for vehicular nodes $j$ and $k$, respectively. The time duration for which vehicular node $j$ and vehicular node $k$ will stay in range of each other is given by the Eq. 2. 


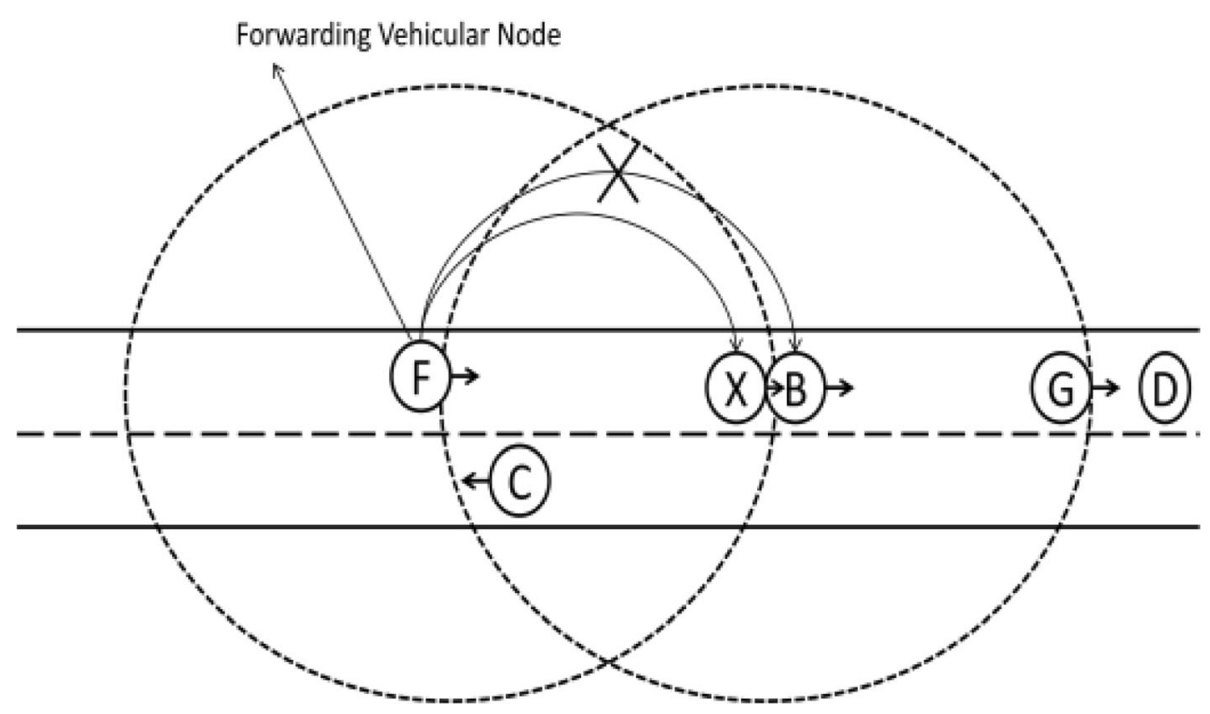

Fig. 4 Limitation of existing forwarding strategies without link stability. $F$ is the forwarding node, $X$ and $B$ are the neighbors that are moving in the direction of destination, and $\mathrm{C}$ is a vehicle traveling in opposite direction to the destination. $\mathrm{D}$ is the destination node. The big dotted circles represent the transmission ranges of the nodes

$$
\mathrm{LDT}=\frac{-(\mathrm{ab}+\mathrm{cd})+\sqrt{\left(\mathrm{a}^{2}+\mathrm{c}^{2}\right) \mathrm{r}^{2}-(\mathrm{ad}-\mathrm{bc})^{2}}}{\mathrm{a}^{2}+\mathrm{c}^{2}}
$$

where

$$
\begin{aligned}
& \mathrm{a}=\mathrm{V}_{\mathrm{k}} \cos \theta_{\mathrm{k}}-\mathrm{V}_{\mathrm{j}} \cos \theta_{\mathrm{j}} \\
& \mathrm{b}=\mathrm{X}_{\mathrm{k}}-\mathrm{X}_{\mathrm{j}} \\
& \mathrm{c}=\mathrm{V}_{\mathrm{k}} \sin \theta_{\mathrm{k}}-\mathrm{V}_{\mathrm{j}} \sin \theta_{\mathrm{j}} \\
& \mathrm{d}=\mathrm{Y}_{\mathrm{k}}-\mathrm{Yj} .
\end{aligned}
$$

In the aforementioned formula, LDT represents the link duration time between the nodes; $V_{j}$ and $V_{k}$ are the velocities of the packet carrier or forwarding node and next candidate receiver node, respectively. The transmission range of the wireless vehicular node is given by $r$. Theta $(\theta)$ represents the direction of movement of the nodes with respect to each other. $\left(X_{j}, Y_{j}\right)$ and $\left(X_{k}, Y_{k}\right)$ are the coordinates of the packet sender and receiver, respectively. Factor $a$ represents the relative velocity of the candidate receiver node with respect to packet sender node along the $Y$-axis. Factor $b$ represents the distance of the receiver node from the packet sender node along the $X$-axis. The relative velocity of the receiver node with respect to the packet sender node along the $Y$-axis is represented by factor $c$. The distance of the receiver node from the sender node along with the $Y$-axis is represented by factor $d$.

In order to discard selection of a node that is about to leave the range of the sender until the packet is delivered to that node, as a first simple step, let us simplify the communication model to get a rough estimate about the time required for transmission and receiving of a packet of size "s" kilobytes. There are two different things to consider:

1) The transmission time

This is the amount of time from the beginning until the end of a message transmission. In the case of a digital message, it is the time from the first bit until the last bit of a message has left the transmitting node. The packet transmission time in seconds can be obtained from the packet size in bits and the bit rate in bits/s as packet transmission time $=$ packet size/bit-rate, and both of these units are in bits [45].

\section{2) Propagation delay}

It is the time it takes for the first bit to travel from the sender to the receiver. The distance here is the distance between the sender and the receiver in meters and the propagation speed is the speed of light, i.e., $3 \times 10^{8} \mathrm{~m} / \mathrm{s}$.

Propagation time $=$ distance $/$ propagation speed

3) Packet delivery time (PDT)

The total time it will take for the packet to be completely transmitted from sender node to the receiver node is given by:

Packet delivery time $(\mathrm{PDT})=$ transmission time $(\mathrm{TT})$ + propagation delay (PD)

Any neighbor whose expected link duration time is less than the expected packet delivery time will not be 
considered as the next node. Algorithm 2 decides the neighbor that is chosen as the next forwarding node.

4) Estimation of closeness and direction of motion

The packet carrier vehicle uses the following equation to choose a neighbor vehicle that moves towards the destination and is closest to the destination.

$$
\text { Score }_{\mathrm{i}}=\sigma \times\left(1-\mathrm{Dn}_{\mathrm{i}} / \mathrm{Dcv}\right)+\rho \times \cos \left(\mathrm{Vn}_{\mathrm{i}}, \operatorname{posn}_{\mathrm{i}}, \mathrm{dv}\right)
$$

In the aforementioned Eq. 3 , the first factor $\left(1-\mathrm{Dn}_{\mathrm{i}} / \mathrm{Dcv}\right)$ represents the closeness of a neighbor vehicle to the destination. The second factor represents the moving direction of the vehicular node using cosine values of two vectors (velocity and position). Here, $\sigma$ and $\rho$ are the weighting factors for closest position and direction of the vehicular node with respect to the destination. We can adjust the value of $\sigma$ and $\rho$ to make a tradeoff between position and direction when forwarding. If the value of $\rho$ is set to 0 , then the protocol uses greedy forwarding. On the other hand, if $\sigma$ is set to 0 , then protocol uses directional forwarding which will be unable to select a neighbor node that is the closest to the destination. Therefore, our approach will consider both by setting the value of $\sigma=0.5$ and $\rho=0.5$.

Below, in our reliable packet forwarding algorithm 2 , the packet carrier or forwarding vehicle considers the position, direction, expected packet delivery time, and link duration time for assigning the score to each of candidate neighboring nodes. It assigns the higher weighting score to those neighbors that are the closest to the destination and moving towards the destination that are capable of successfully receiving the message based on link duration time. In our algorithm, the current packet carrier vehicular node uses line 8-17 for determining its next candidate neighbor. In line 15 , the forwarding vehicle compares each neighbor link duration time with expected packet delivery time. For any neighbor, if link duration time is less then packet delivery

Algorithm 2: The Reliable Packet Forwarding Mechanism

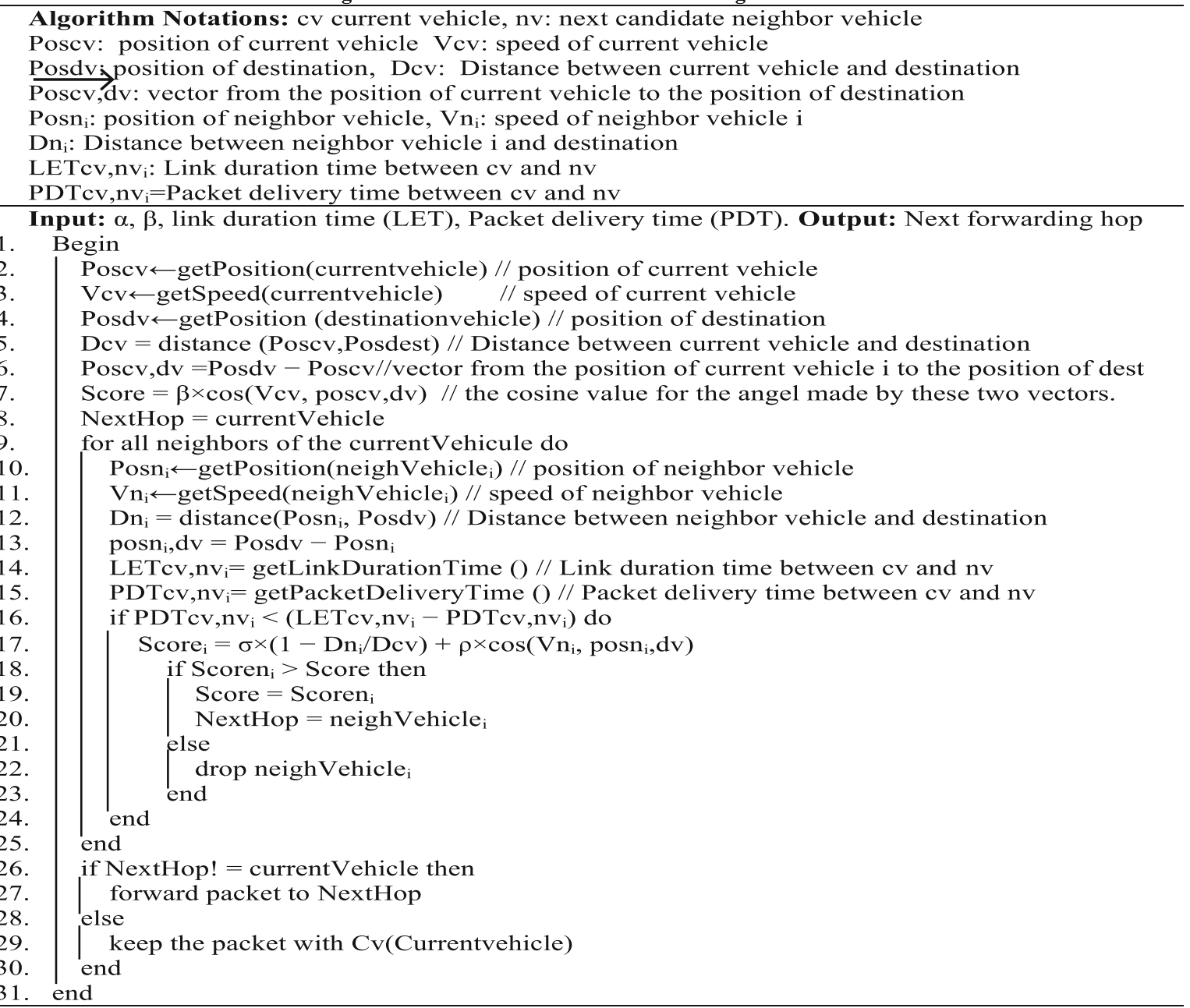


time or not satisfying the condition mentioned in line 15 will be not be considered. On the other hand, if link duration time is greater than expected packet delivery time and satisfying the equation mentioned in line 13 , then a score is assigned according to the equation mentioned in line 13 . This equation considers the position as well as the direction of motion of the neighbors. Among all the neighbors, the neighbor that is closest to the destination and moving in the direction of destination is assigned highest weighting score and is selected as the next forwarding vehicle. $\sigma$ and $\rho$ are the weighting factors for the closeness of the next hop neighbor and its movement direction with respect to destination respectively. Their value is set to $\sigma=\rho=0.5$. Sometimes a packet carrier vehicle is unable to locate a next candidate forwarding vehicle to forward the packet. In this case, the packet carrier vehicle will hold the packet until next intersection or an appropriate forwarder node in its vicinity is found.

\section{Simulation and results analysis}

In this section, we evaluate the performance of RPSPF. The simulations are carried out in ONE [19] simulator. The mobility model can affect the traffic characteristics which affects the performance of a routing protocol. Therefore, the selection of an appropriate realistic mobility model for generating realistic mobility traces for simulations is a very vital step [46]. We used SUMO (simulation of urban mobility) [22] to generate the realistic vehicular mobility patterns. It is a microscopic road traffic simulation package which is open source. The realistic mobility traces generated by SUMO include all essential characteristics of city environment like multiple lane roads, communication obstacles, and vehicle speed regulations in accordance with traffic signals at intersections.

\subsection{Simulation setup}

The simulation scenario comprises of $3000 \times 2500 \mathrm{~m}^{2}$ city area with 32 multilane bidirectional roads and 32 intersections. At the start of the simulations, all the vehicular nodes were positioned randomly over the multilane bidirectional roads. The movement of the vehicular nodes in both directions on a multilane road is based on the intelligent driving model [22]. The simulation parameters that are used for performance analysis are presented in Table 2. The simulation outcomes are based on an average of 15 simulation runs.

\subsection{Results}

The metrics that are used for evaluation of the routing protocols include the packet delivery ratio, end-to-end delay, and routing overhead. Packet delivery ratio is the fraction of packets that are effectively dispatched to their destination vehicular nodes. End-to-end delay is the average delay incurred by a packet while moving from its source to destination. While routing overhead is the fraction of total control packets generated to the total data packets delivered at destinations during one complete simulation run. The detailed depiction of the aforementioned metrics is provided in $[1,11,14,36,47]$. We provide a comparative study and in-depth analysis of RPSPF with respect to GPSR, GSR, GyTAR, and TFOR considering the aforementioned metrics.

\subsubsection{Packet delivery ratio}

Figure 5 presents the impact of increasing node density on the packet delivery ratio. In Fig. 5, it is illustrated that as the vehicular density increases, the packet delivery ratio of all the considered routing protocols increases. The figure exhibits that the packet delivery ratio of the RPSPF is the highest as compared to GPSR, GSR, E-GyTAR, and TFOR. This is because of two reasons, firstly, in RPSPF, the routing path is set up based on multiple intersections guaranteeing that the intersections with rich traffic density are chosen. Therefore, the packet will move successively towards destination along the streets which contains enough vehicles ensuring rich network connectivity. Secondly, RPSPF provides a reliable link stability mechanism that ensures that before forwarding the data packet, the link has enough life-time

Table 2 Simulation setup

\begin{tabular}{llll}
\hline Simulation/scenario & & MAC/routing & \\
\hline Simulation time & $250 \mathrm{~min}$ & MAC protocol & 802.11 DCF \\
Map size & $3000 \times 2500 \mathrm{~m}^{2}$ & Channel capacity & $54 \mathrm{Mbps}$ \\
Mobility model & $\mathrm{SUMO}$ & Transmission range & $266 \mathrm{~m}$ \\
Number of intersections & 32 & Traffic model & 15 CBR connections \\
Number of double lane roads & 36 & Packet sending rate & $(1-10$ packet(s)/s) \\
Number of vehicles & $100-400$ & Vehicle speed & $35-60 \mathrm{Km} / \mathrm{h}$ \\
Weighting factors & $a=0.5, \beta=0.5$, & Beacon interval & $1 \mathrm{~s}$ \\
& $H_{1}=0.5, \mathrm{H}_{2}=0.5$ & & \\
\hline
\end{tabular}




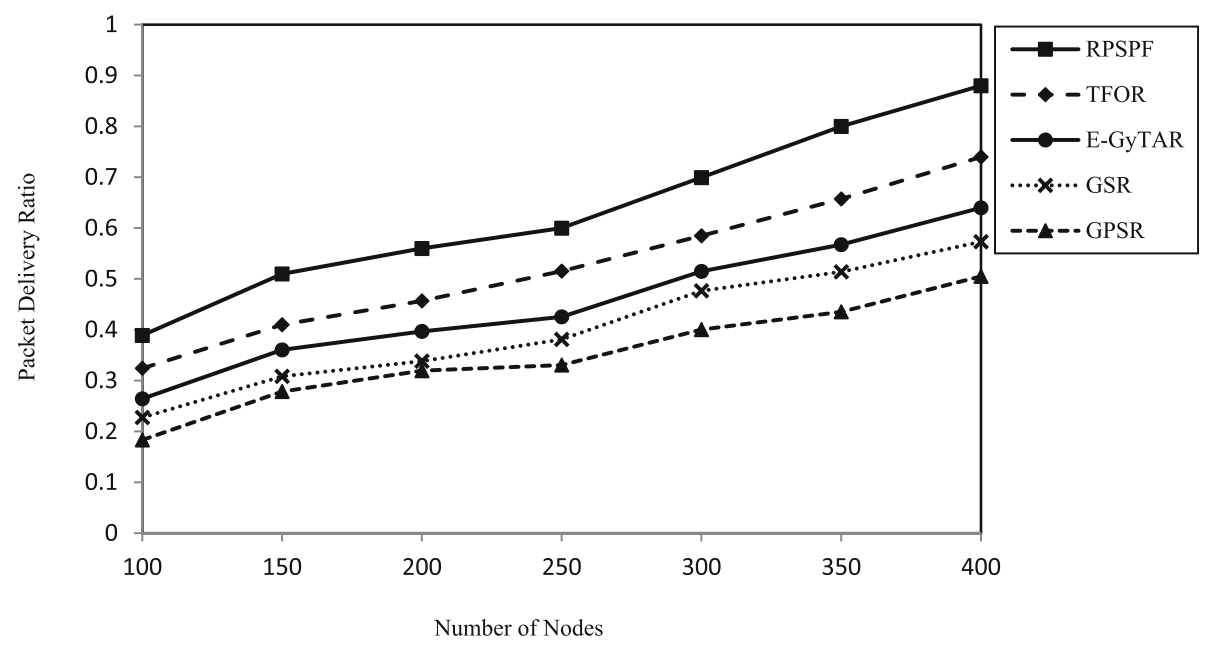

Fig. 5 Packet delivery ratio as a function of the number of nodes (@5 packets/s). RPSPF represents Reliable Path Selection and Packet Forwarding Routing Protocol which is proposed approach and is represented by a solid line with a square. TFOR is traffic flow-oriented routing protocol and is represented by a dashed line having rhombus. E-GyTAR is Enhanced Greedy Traffic Aware Routing protocol and is represented by a solid line with circle. GSR is geographic source routing protocol and is presented by a dotted line having a cross. GPSR is Greedy Perimeter Stateless Routing Protocol and is presented by a dashed line having a triangle

to accomplish successful forwarding. This avoids link ruptures before a packet is completely delivered to the destination. GSR computes a sequence of intersections statically without considering vehicular traffic density. As a result, sometimes it selects routing paths that consist of city streets with low vehicular traffic density. As a result, packets are unable to move towards destination due to lack of connectivity which degrades the packet delivery ratio. E-GyTAR and TFOR accomplish routing path dynamically based on one intersection at a time. Dynamically, considering one intersection at a time might lead to a selection of those intersections whose next streets contain no or very low vehicular traffic. Selection of such streets results increases the probability of encountering local optimum at street level which leads to a reduction in packet delivery ratio. Also, GSR, EGyTAR, and TFOR protocols during forwarding mechanism may select those neighbor nodes which are the closest to the destination but their link duration time is not enough to get packets successfully transferred from the forwarding nodes. In other words, the links break before successful delivery of the packets and packets are lost which also degrades the packet delivery ratio.

Figure 6 presents the impact of packet sending rate on the packet delivery ratio. Increase in packet sending rate causes network congestion and packets collision which brings down the packet delivery ratio. It affects the performance of all the routing protocols. Some vehicular nodes along the preselected routing paths in GSR transmit more control messages due to static intersection selection mechanism which causes a reduction in the packet delivery ratio as compared to RPSPF, E-GyTAR, and TFOR. Unreliable forwarding mechanism in GSR, GPSR, E-GyTAR, and TFOR also affects the packet delivery ratio because more packets are re-generated and dropped due to link ruptures.

Figure 7 illustrates the influence of increasing file size (data size) on packet delivery ratio. If we increase the file size, the packet delivery ratio tends to decrease for all the considered protocols. This is because, in VANETs, due to the high mobility of nodes, the topological connections are ephemeral. More time is required for transfer of files having a larger size as compared to smaller

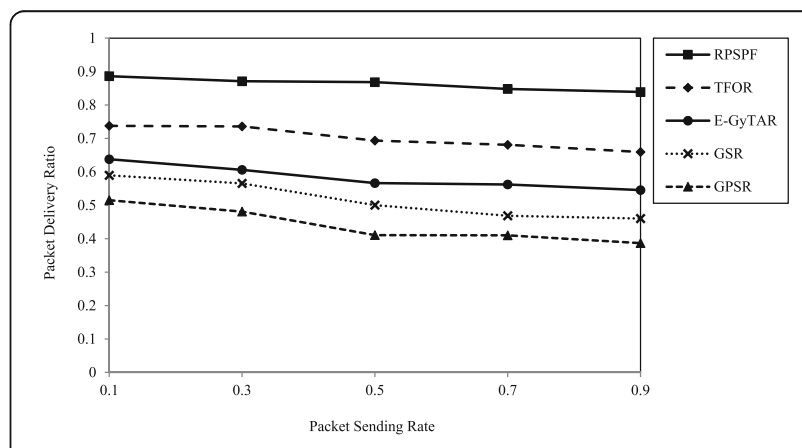

Fig. 6 Packet delivery ratio as a function of the packet sending rate (400 nodes). RPSPF represents Reliable Path Selection and Packet Forwarding Routing Protocol which is proposed approach and is represented by a solid line with a square. TFOR is traffic floworiented routing protocol and is represented by a dashed line having rhombus. E-GyTAR is Enhanced Greedy Traffic Aware Routing protocol and is represented by a solid line with circle. GSR is geographic source routing protocol and is presented by a dotted line having a cross. GPSR is Greedy Perimeter Stateless Routing Protocol and is presented by a dashed line having a triangle 


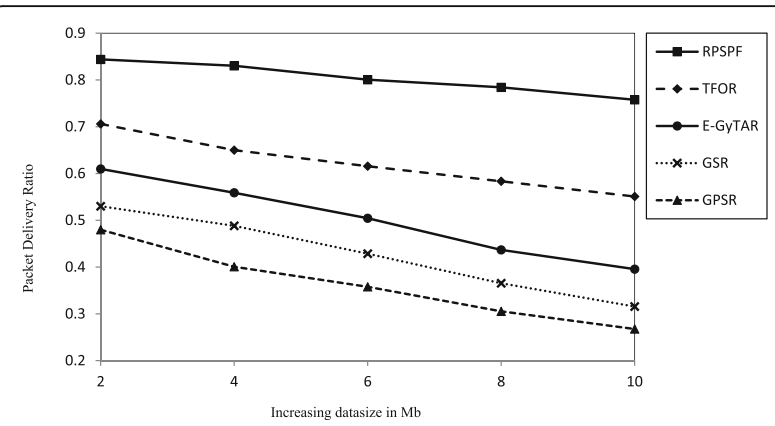

Fig. 7 Influence of increasing data size on packet delivery ratio (400 nodes). RPSPF represents Reliable Path Selection and Packet Forwarding Routing Protocol which is proposed approach and is represented by a solid line with a square. TFOR is traffic floworiented routing protocol and is represented by a dashed line having rhombus. E-GyTAR is Enhanced Greedy Traffic Aware Routing protocol and is represented by a solid line with circle. GSR is geographic source routing protocol and is presented by a dotted line having a cross. GPSR is Greedy Perimeter Stateless Routing Protocol and is presented by a dashed line having a triangle

files. Choosing a suitable node with sufficient contact duration for forwarding a larger file is of immense importance. It can be observed from the figure that as we increase the file size from 2 to $10 \mathrm{MB}$, the packet delivery ratio degrades for all the protocols. However, our proposed protocol outperforms the other protocols. This is because, firstly, our protocol dispatches the packets towards destination through those streets that maximize connectivity. Secondly and most importantly, the forwarding node always prefers to forward the packet to a neighbor that offers enough link life-time to carry out successful file transfer which decreases the probability of packet loss. The rest of the protocols prove to be ineffective in providing higher packet delivery ratio. The major reason behind this is that their forwarding strategies look to forward the packet to a node without considering link lifetime. The links often break before the successful transfer of the file due to the short link lifetime. As a result, packet loss increases and this leads to decrease in packet delivery ratio.

\subsubsection{End-to-end delay}

Figure 8 exhibits the performance of RPSPF, E-GyTAR, GPSR, GSR, and TFOR in terms of end-to-end delay with respect to increasing traffic density. RPSPF outperforms GPSR, GSR, E-GyTAR, and TFOR in terms of end-to-end delay as well. This is because, RPSPF progressively accomplishes routing path based on multiple intersection selection mechanism by considering network connectivity when relaying data packets from source to destination. The reliable greedy forwarding mechanism maintaining one-hop information based on link stability accomplishes successful forwarding avoids packet retransmission and helps to reduce the end-to-

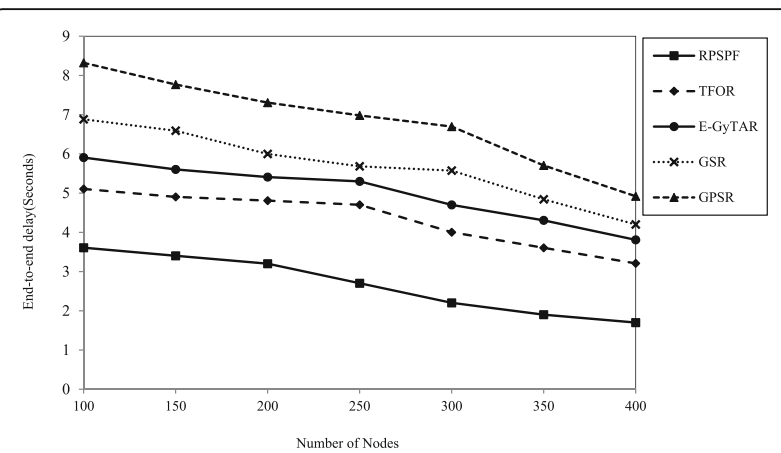

Fig. 8 End-to-end delay as a function of the number of nodes (@5 packets/s). RPSPF represents Reliable Path Selection and Packet Forwarding Routing Protocol which is proposed approach and is represented by a solid line with a square. TFOR is traffic floworiented routing protocol and is represented by a dashed line having rhombus. E-GyTAR is Enhanced Greedy Traffic Aware Routing protocol and is represented by a solid line with circle. GSR is geographic source routing protocol and is presented by a dotted line having a cross. GPSR is Greedy Perimeter Stateless Routing Protocol and is presented by a dashed line having a triangle

end delay as well. While in E-GyTAR and TFOR, their intersection selection mechanisms sometimes compel them to select those routing paths that have negligible connectivity; therefore, a packet stays the longer time in a buffer which results in long delays. Also, E-GyTAR uses only directional density to find the path but in urban scenarios with a two-lane road, there are a lot of streets having the non-directional density for providing the shortest path which avoids end-to-end delay. Pre-determination of end-to-end path routing path in GSR before dispatching data packets without considering connectivity causes delays due to lack of traffic density along some routes. In GPSR, perimeter phase establishes longer routes while relaying the packet towards the destination. It also causes routing loops which results in long delays. The novel combination of multiple intersection selection mechanism with reliable forwarding mechanism in RPSPF leads to a considerable reduction of end-to-end delay in comparison to the other protocols.

Figure 9 provided end-to-end delay with respect to different packet sending rates on the logarithmic scale. It shows that an increase in packet sending rate does not have a considerable impact on the performance of RPSPF as compared to the considered routing techniques. This is because of its new intersection selection mechanism and reliable forwarding which can incorporate the city challenges like high mobility and finding streets which provide better connectivity for routing in a better way as compared to earlier techniques.

\subsubsection{Routing overhead}

Routing overhead with respect to a different number of the nodes for all the routing protocols is illustrated in 


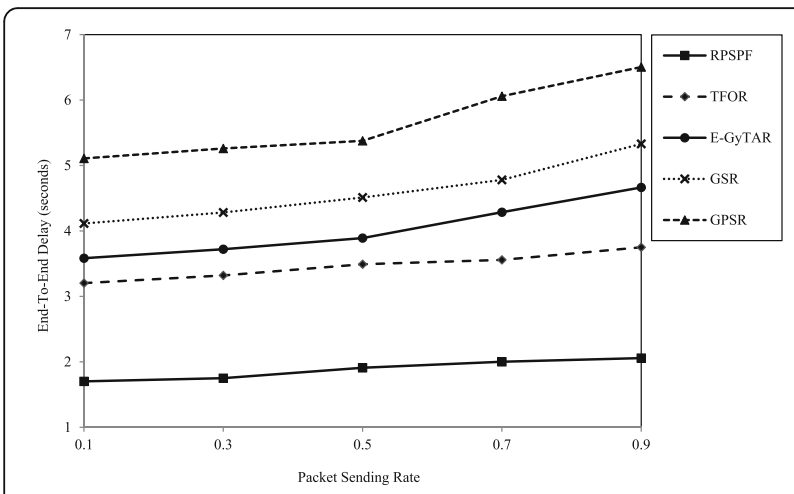

Fig. 9 End-to-end delay as a function of the packet sending rate (400 nodes). RPSPF represents Reliable Path Selection and Packet Forwarding Routing Protocol which is proposed approach and is represented by a solid line with a square. TFOR is traffic floworiented routing protocol and is represented by a dashed line having rhombus. E-GyTAR is Enhanced Greedy Traffic Aware Routing protocol and is represented by a solid line with circle. GSR is geographic source routing protocol and is presented by a dotted line having a cross. GPSR is Greedy Perimeter Stateless Routing Protocol and is presented by a dashed line having a triangle

Fig. 10. An increase in traffic density increases routing overhead for all the protocols. This is because the number of control messages produced during simulation is directly proportional to the number of vehicles. The routing overhead incurred by RPSPF is least as compared to the other routing protocol. For acquiring neighbor locations, GSR produces more beacon messages which increase its routing overhead. The number of beacon messages generated by GSR is three times more as compared to E-GyTAR [1]. GPSR recovery strategy incurs more packet transmission which induces higher

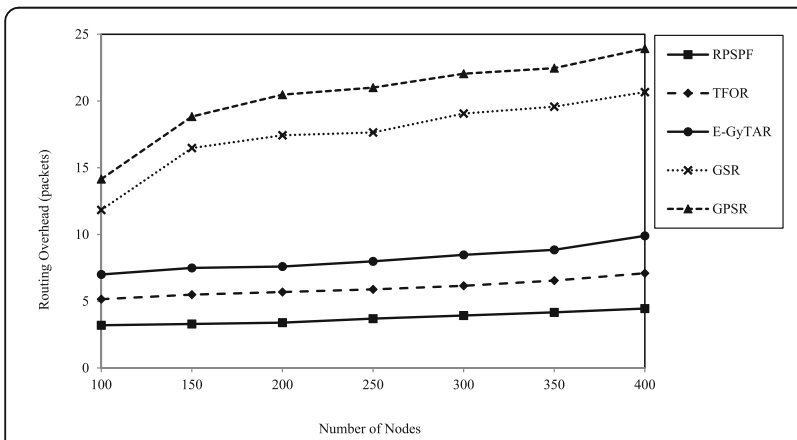

Fig. 10 Routing overhead as a function of the number of nodes (in 5 packet/s). RPSPF represents Reliable Path Selection and Packet Forwarding Routing Protocol which is proposed approach and is represented by a solid line with a square. TFOR is traffic floworiented routing protocol and is represented by a dashed line having rhombus. E-GyTAR is Enhanced Greedy Traffic Aware Routing protocol and is represented by a solid line with circle. GSR is geographic source routing protocol and is presented by a dotted line having a cross. GPSR is Greedy Perimeter Stateless Routing Protocol and is presented by a dashed line having a triangle routing overhead. While both E-GyTAR and TFOR also incur more routing overhead due to inappropriate intersection selection mechanism and unreliable forwarding strategies. Packets are lost due to sudden link ruptures while forwarding which results in retransmission of the packets resulting in an increase in routing overhead. Also, maintenance of two-hop neighbor information in TFOR causes more routing overhead in high-traffic density scenarios like traffic jams. As shown in Figs. 5 and 6 , the packet delivery ratio for the proposed protocol is better as compared to all the other considered protocols; therefore, it means that lesser packets need to be transmitted in case of the proposed protocol resulting in decreased routing overhead. Forwarding mechanism without link reliability causes packet loss and results in increased routing overhead for all the routing protocols.

Figure 11 shows the routing overhead with respect to the different packet sending rates. The increase in packet sending rate increases the routing overhead for all the considered protocols. The proposed protocol is least affected with respect to an increase in packet sending rate. The major reason behind this is that due to the enhancement that the proposed protocol brings, the packet delivery ratio is higher and the number of packets lost is lower. As a result, increase in packet sending rate does not substantially degrade the performance of the proposed routing protocol as compared to the other protocols.

\subsubsection{Influence on performance by increasing the number of considered intersections dynamically}

One essential query that needs to be explored is that what is the optimal number of intersections that should

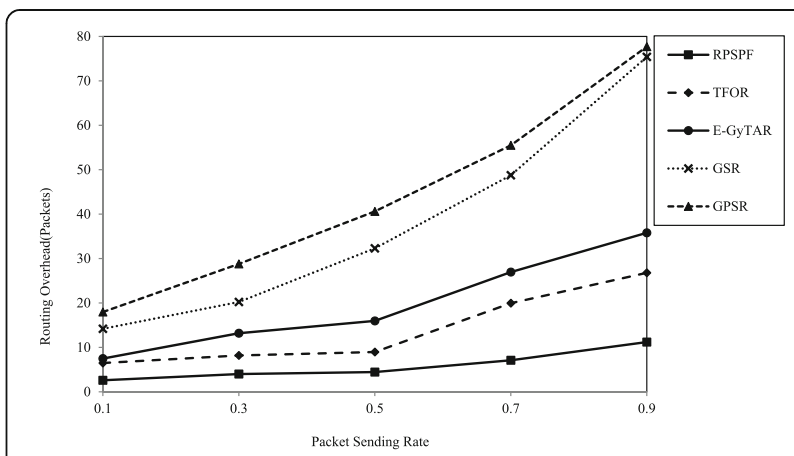

Fig. 11 Routing overhead as function of packet sending rate (400 nodes). RPSPF represents Reliable Path Selection and Packet Forwarding Routing Protocol which is proposed approach and is represented by a solid line with a square. TFOR is traffic floworiented routing protocol and is represented by a dashed line having rhombus. E-GyTAR is Enhanced Greedy Traffic Aware Routing protocol and is represented by a solid line with circle. GSR is geographic source routing protocol and is presented by a dotted line having a cross. GPSR is Greedy Perimeter Stateless Routing Protocol and is presented by a dashed line having a triangle 
be considered for accomplishing the best performance? For responding such query, we have compared the performance in terms of packet delivery ratio with respect to increasing the number of considered intersections. The outcome of simulation depicts that consideration of two intersections gives us the better performance as compared to increasing intersection beyond two.

According to Fig. 12, increasing the number of considered intersections from 1 to 2 gives us an increase in packet delivery ratio. On the other hand, as we begin to increase the number of intersection beyond 2, RPSPF performance begins to decline in terms of packet delivery ratio. The primary reason behind this study lies in one of the very fundamental characteristics of VANETs, i.e., the VANETs have a very dynamic network in nature and its topology alters very quickly. For this reason, considering more than two intersections while deciding for packet forwarding degrades the performance instead of accomplishing an enhanced performance. Furthermore, maintaining all the information that lies between multiple intersections, such as traffic density and vehicle direction, in a highly dynamic topology also direct to an increased overhead in terms of processing and storage.

The simulation outcomes show that our protocol RPSPF that contains dynamic multiple intersections selection mechanism with reliable forwarding brings the considerable improvement over other routing approaches. It enhances the performance of the network by providing paths with better connectivity and forwarding packets through stable links. The novel mechanisms introduced in RPSPF lead to performance enhancement in terms of end-to-end delay, packet delivery ratio, and routing overhead.

\section{Conclusions}

In this paper, we have presented a new reliable path selection packet forwarding protocol for VANETs called

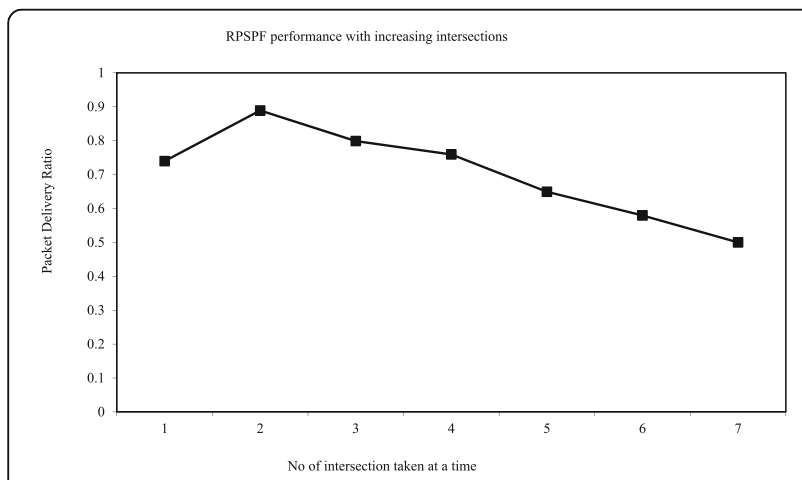

Fig. 12 Packet delivery ratio vs increasing number of intersections dynamically at a time in 400 nodes. RPSPF indicates Reliable Path Selection and Packet Forwarding Routing Protocol which is the proposed approach. It is presented in the figure with a solid line having a square as Reliable Path Selection and Packet Forwarding Routing Protocol (RPSPF). At the beginning, we have discussed a detailed technical analysis and comparison of the existing state-of-the-art routing strategies by highlighting the major limitations countenanced by these approaches. After that, we provided details of our proposed routing technique and explained how it overcomes the limitations of existing techniques. RPSPF chooses a couple of intersections at a time on the basis of the shortest curve metric distance to the target node and vehicular traffic density. RPSPF make use of a new multiple intersections selection mechanism, a novel reliable and stable greedy forwarding approach to relay packets in between intersections, and a recovery technique, which is capable of incorporating city environments more efficiently. Simulation results have revealed that RPSPF surpasses TFOR, E-GyTAR, GPSR, and GSR in terms of various metrics like packet delivery ratio, end-to-end delay, and routing overhead. This is due to its ability of incorporating city surroundings' main challenges to routing in a better way as compared to earlier routing techniques.

\section{Abbreviations}

ADASE2: Advanced driver-assistance systems; AODV: Ad hoc On-Demand Distance Vector; A-STAR: The Anchor Based Street and Traffic Aware; CAMP: Crash Avoidance Metrics Partnership; DGR: Directional Greedy Routing Protocol; DGSR: Directional Greedy Source Routing; DSR: Dynamic Source Routing; DSRC: Dedicated short-range communication; E-GyTAR: Enhanced Greedy Traffic Aware Routing; E-GyTARD: Enhanced Greedy Traffic Aware Routing Directional; E-GyTAR-PD: Enhanced Greedy Traffic Aware Predictive Directional; GLS: Grid Location Service; GPCR: Greedy Perimeter Coordinator Routing; GPS: Global Positioning System; GPSR: Greedy Perimeter Stateless Routing; GSR: Geographic source routing; GyTAR: Greedy Traffic Aware Routing; IFTIS: Infrastructure Free Traffic Information System; ITS: Intelligent Transportation System; LDT: Link duration time; MANET: Mobile ad hoc network; OLSR: Optimized Link State Routing; PD: Propagation delay; PDGR: Predictive Directional Greedy Routing; PDT: Packet delivery time; PDVR: Predictive Directional Vehicular Routing; RPSPF: Reliable Path Selection Packet Forwarding; SUMO: Simulation of urban mobility; TFOR: Traffic flow-oriented routing protocol; TT: Transmission time; VANET: Vehicular ad hoc networks

\section{Acknowledgements}

The first author (the main author) acknowledges the useful feedback provided by the reviewers for improving this article. The main author also acknowledges the financial assistance and support provided during this research work by the Universiti of Malaysia Sarawak.

\section{Funding}

The work is fully funded by the Research and Innovation Management Center University of Malaysia Sarawak (RIMC-UNIMAS) under the grant number F08/SpSG/1403/16/4.

Availability of data and materials

All relevant data are within the paper and its supporting information files.

Authors' contributions

IAA is the main author of this manuscript. He conceived the novel ideas, designed the algorithms and experiments, and performed the analysis. He wrote the entire manuscript. He accomplished all the revisions provided during entire peer review process until publication. He conducted the final proof reading as well. This manuscript is the outcomes of the reseach activities carried out only by the main author. IAA, ASK and SA checked and reviewed the manuscript. ASK supervised the work. All authors have read and approved the final manuscript. 


\section{Authors' information}

Irshad Ahmed Abbasi is working as a researcher at the Department of Computer Science and Information Technology, Universiti Malaysia Sarawak, Malaysia. He served as a senior lecturer at King Khalid University, Kingdom of Saudi Arabia. He is also a senior lecturer at the Department of Computer Science, University of Bisha, Kingdom of Saudi Arabia. He has over ten years of research, and teaching experience. Abbasi has received multiple awards, scholarships, and research grants. His research interests include routing in VANETs and MANETs, Mobile Computing, loT, and Cloud Computing. He is an author of many articles including Traffic Flow Oriented Routing Protocol for VANETs, published in Springer Eurasip Journal on Wireless Communication and Networking. He is also acting as a reviewer of many well reputed peer reviewed international journals and conferences.

Adnan Shahid Khan is currently a senior lecturer at Faculty of Computer Science and Information Technology, Universiti Malaysia Sarawak. He has completed his Postdoctoral, PhD, and Masters in Networks in 2013, 2012, and 2008, respectively, from Universiti Teknologi Malaysia, Johor Bahru, Malaysia, and BSc (Hons) Computer Sciences in 2005 from University of Punjab, Lahore Pakistan. His research interest includes Network and Network security in Wireless communication. He has published more than 60 papers in peer reviewed international conferences and journals.

Shahzad Ali received his M.Sc. in telematics engineering in 2011 and his Ph.D. in telematics engineering from University Carlos III of Madrid in 2014. Currently, he is working as an assistant professor at COMSATS Institute of Information Technology, Abbottabad, Pakistan. His research interests include performance analysis of context-aware applications, wireless sensor networks, vehicular ad hoc networks, and opportunistic networks. He has published many articles in peer reviewed international conferences and journals.

\section{Competing interests}

The authors declare that they have no competing interests.

\section{Publisher's Note}

Springer Nature remains neutral with regard to jurisdictional claims in published maps and institutional affiliations.

\section{Author details}

'Department of Computer Science and Information Technology, Universiti Malaysia Sarawak (UNIMAS), 9300 Kota Samarahan, Malaysia. ${ }^{2}$ Department of Computer Science, Faculty of Science and Arts at Balgarn, University of Bisha, P.O. Box 60, Sabt Al-Alaya 61985, Kingdom of Saudi Arabia. ${ }^{3}$ Department of Computer Science, COMSATS Institute of Information Technology, Abbottabad, Pakistan.

Received: 16 October 2017 Accepted: 27 August 2018 Published online: 10 October 2018

\section{References}

1. S.M. Bilal, S.A. Madani, I.A. Khan, Enhanced junction selection mechanism for routing protocol in VANETs. Arab J. Inf. Technol. 8(4), 422-429 (2011)

2. G.M.T. Abdalla, M.A.A. Rgheff, S.M. Senouci, Current trends in vehicular ad hoc networks. UbiCC J. Special Issue UbiRoads 10(21), 1-14 (2008)

3. K.C. Lee, U. Lee, M. Gerla, Advances in Vehicular Ad-Hoc Networks: Developments and Challenges, chapter Survey of Routing Protocols in Vehicular Ad Hoc Networks (IGI Global, Hershey, 2009)

4. Car-talk 2000. http://cartalk2000.net.outerstats.com/. Accessed 10 May 2013

5. CAR 2 CAR Communication Consortiums. https://www.car-2-car.org/index. php?id=5. Accessed 2 June 2011

6. California Partners for Advanced Transit and Highways. https://path.berkeley. edu. Accessed 15 May 2011

7. FleetNet. https://fleetnetamerica.com. Accessed 17 Oct 2009

8. N. Gupta, A. Prakash, R. Tripathi, Adaptive beaconing in mobility aware clustering based MAC protocol for safety message dissemination in VANET, Hindawi Publishing Corporation. Wireless Commun. Mob. Comput. 5(4), 1-15 (2017)

9. Chauffeur in EU. https://www.autoeurope.com/chauffeur-services/. Accessed 7 Sept 2015

10. Crash Avoidance Metrics Partnership. https://www.mentor.com. Accessed 5 Feb 2005
11. I.A. Abbasi, B. Nazir, A. Abbasi, S.M. Bilal, S.A. Madani, A traffic flow oriented routing protocol for VANET. Springer EURASIP I Wireless Commun Netw 2014(121), 1-14 (2014)

12. B.T. Sharef, R.A. Alsaqour, M. Ismail, Review: vehicular communication ad hoc routing protocols: a survey. J. Netw. Comput. Appl. 5(4), 363-396 (2014)

13. A. Dahiya, R.K. Chauhan, A comparative study of MANET and VANET environment. J. Comput. Secur. 2(7), 87-91 (2010)

14. M. Jerbi, S.M. Senouci, R. Meraihi, Y.G. Doudane, in Communications, 2007. ICC '07. IEEE International Conference. An improved vehicular ad hoc routing protocol for city environments (2007), pp. 3972-3979

15. J. Kumar, V. Mutneja, I.S. Gill, Behavior of position based routing in VANET. Int. J. Comput. Appl. 145(1), 49-52 (2016)

16. Y.W. Lin, Y.S. Chen, S.L. Lee, Routing protocols in vehicular ad hoc networks: a survey and future perspectives. J. Inf. Sci. Eng. 26(3), 913-932 (2010)

17. H. Hartenstein, K.P. Laberteaux, VANET Vehicular Applications and Inter-networking Technologies, 1st edn. (Wiley Online Library, United Kingdom, UK, 2010)

18. K. Prasanth, K. Duraiswamy, K. Jayasudha, C. Chandrasekar, K. Prasanth, K. Duraiswamy, K. Jayasudha, C. Chandrasekar, Improved packet forwarding approach in vehicular ad hoc networks using RDGR algorithm. Int. J. Next Gene. Netw. (IJNGN) 2(1), 64-77 (2010)

19. A. Keranen, J. Ott, T. Karkkainen, in Proceedings of the 2nd International Conference on Simulation Tools and Techniques. The ONE simulator for DTN protocol evaluation (2009)

20. S Dhankhar, S AgrawalA survey on routing protocols and issues. International Journal of Innovative Research in Science, Eng. Technol. 3(6), 1-14(2014)

21. J. Cheng, J. Cheng, M.C. Zhou, F.Q. Liu, S.C. Gao, C. Liu, Routing in internet of vehicles: a review. IEEE Transac. Intel. Transport. Syst. 16(5), 1-15 (2015)

22. M. Behrisch, L. Bieker, J. Erdmann, D. Krajzewicz, in International Conference on Advances in System Simulation. SUMO-simulation of urban mobility (2011), pp. 63-68

23. D.B. Johnson, D.A. Maltz, in Mobile Computing. Dynamic source routing in ad hoc wireless networks (Kluwer Academic Publishers, Alphen aan den Rijn, 1996), pp. 153-181

24. CE Perkins, EM Royer, Ad-hoc on-demand distance vector routing, in Mobile Computing Systems and Applications, 1999. Proceedings. WMCSA '99. Second IEEE Workshop, 1999, pp. 90-100

25. T. Clausen, P. Jacquet, Optimized Link State Routing Protocol (OLSR), RFC Editor, United States, 2003. https://doi.org/10.17487/RFC3626

26. S. Jaap, M. Bechler, L. Wolf, Evaluation of Routing Protocols for Vehicular Ad Hoc Networks in City Traffic Scenarios, in Proceedings of the 5th International Conference on Intelligent Transportation Systems Telecommunications (ITST) (Brest, France, 2005)

27. F. Li, Y. Wang, Routing in vehicular ad hoc networks: a survey. Vehic Technol Magaz IEEE 2(2), 12-22 (2007)

28. B Karp, HT Kung, GPSR: greedy perimeter stateless routing for wireless networks, in Proceedings of the 6th Annual International Conference on Mobile Computing and Networking, MobiCom '00 (ACM, New York, NY, USA, 2000), pp. 243-254

29. D Tian, K Shafiee, VCM Leung Position-based directional vehicular routing, in Proceeding of the Global Telecommunications Conference, GLOBECOM (IEEE, Hoboken, 2009), pp.1-6

30. B.C. Seet, G. Liu, B.S. Lee, C.H. Foh, K.J. Wong, K.K. Lee, in Lecture Notes in Computer Science: NETWORKING 2004, Networking Technologies, Services, and Protocols; Performance of Computer and Communication Networks; Mobile and Wireless Communications. A-STAR: a mobile ad hoc routing strategy for metropolis vehicular communications (2004), pp. 989-999

31. R. Sharma, A. Choudhry, An extensive survey on different routing protocols and issue in VANETs. Int. J. Comput. Appl. 106(10), 1-16 (2014)

32. Shah et al., Unicast routing protocols for urban vehicular networks: review, taxonomy, and research issues. J Zhejiang Univ-SCIENCE (Computers \& Electronics). 15(7) 489-513 (2014)

33. I.A. Abbasi, A.S. Khan, A Review of Vehicle to Vehicle Communication Protocols for VANETs in the Urban Environment. MDPI J. Future Internet 10(2), 1-14 (2018)

34. A. Srivastava, B.P. Chaurasia, Survey of routing protocol used in vehicular ad hoc networks. Int. J. Curr. Eng. Technol. 7(3), 1-12 (2017)

35. A. Husain, R. Shringar, B. Kumar, A. Doegar, Performance comparison topology based and position based routing protocols in vehicular network environments. Int. J. Wireless Mob. Netw. (IJWMN) 3(4), 1-13 (2011)

36. S.M. Bilal, A.R. Khan, S. Ali, Review and performance analysis of position based routing protocols. Springer Wireless Person. Area Commun. 16(73), 1-13 (2016) 
37. C Lochert, H Hartenstein, J Tian, H Füßler, D Hermann, M Mauve, A routing strategy for vehicular ad hoc networks in city environments, in Proceedings of the Intelligent Vehicles Symposium, 2003. IEEE, 2003, pp. 156-161

38. C Lochert, M Mauve, H Füßler, H Hartenstein, Geographic routing in city scenarios. SIGMOBILE Mob. Comput. Commun. Rev. 9, 69-72 (2005)

39. W. Kieb, H. Fubler, J. Widmer, M. Mauve, Hierarchical location service for mobile ad-hoc networks: SIGMOBILE Mobile. Comput. Commun. Rev. 1(2), 47-58 (2004)

40. M. Kasemann, H. Fubler, H. Hartenstein, M. Mauve, A reactive location service for mobile ad hoc networks, Technical Report, TR-14-2002. Dept. of Computer Science, University of Mannheim; 2002. http://citeseerx.ist.psu.edu/viewdoc/ download;jsessionid=64A84973DF600096DE16D8801C37B86E?doi=10.1.1.12. $8778 \&$ rep $=$ rep $1 \&$ type $=$ pdf

41. J Gong, CZ Xu, J Holle, Predictive directional greedy routing in vehicular ad hoc networks, in Distributed Computing Systems Workshops, 2007. ICDCSW '07. 27th International Conference on June 2007

42. J. Li, J. Jannotti, D.S.J. Decouto, D.R. Karger, R. Morris, in Proceedings of the 6th Annual International Conference on Mobile Computing and Networking, MobiCom '00. A scalable location service for geographic ad hoc routing (ACM, New York, NY, USA, 2000), pp. 120-130

43. M. Jerbi, S.M. Senouci, T. Rasheed, Y. Ghamri, in Proceedings of the First IEEE International Symposium on Wireless Vehicular Communication WiVec '07. An infrastructure free intervehicular communication based traffic information (Baltimore, USA, 2007), pp. 1-10

44. W. Su, S.J. Lee, M. Gerla, Mobility prediction and routing in ad hoc wireless networks. Int. J. Netw. Manag. 11(1), 3-30 (2001)

45. K.R. Kumar, VANETs parameters and applications: a review. Glob. J. Sci. Technol. (GJCST) 10(7), 1-6 (2010)

46. M.F. Politecnico, C.D.D. Abruzzi, J. Harri, F. Filali, Vehicular mobility simulation for VANETS, Christian Bonnet Institut Eurecom, Department of Mobile Communications 06904, Sophia, France (2011), pp. 1-14

47. S. Ali, G. Rizzo, V. Mancuso, M.A. Marsan, in IEEE Conference on Computer Communications (INFOCOM). Persistence and availability of floating content in a campus environment (2015), pp. 2326-2334

\section{Submit your manuscript to a SpringerOpen ${ }^{\circ}$ journal and benefit from:}

- Convenient online submission

- Rigorous peer review

- Open access: articles freely available online

- High visibility within the field

- Retaining the copyright to your article

Submit your next manuscript at $\boldsymbol{\nabla}$ springeropen.com 